\title{
EXISTENCE AND GLOBAL EXPONENTIAL STABILITY OF A PERIODIC SOLUTION TO DISCRETE-TIME COHEN-GROSSBERG BAM NEURAL NETWORKS WITH DELAYS
}

\author{
ZhengQiU Zhang AND LiPING WANG
}

\begin{abstract}
By employing coincidence degree theory and using Halanaytype inequality technique, a sufficient condition is given to guarantee the existence and global exponential stability of periodic solutions for the two-dimensional discrete-time Cohen-Grossberg BAM neural networks. Compared with the results in existing papers, in our result on the existence of periodic solution, the boundedness conditions on the activation are replaced with global Lipschitz conditions. In our result on the existence and global exponential stability of periodic solution, the assumptions in existing papers that the value of activation functions at zero is zero are removed.
\end{abstract}

\section{Introduction}

Since 1983, Cohen and Grossberg [2] constructed a kind of simplified neural networks which are now called Cohen-Grossberg neural networks (CGNNS), they have received increasing interesting due to their promising potential applications in many fields such as pattern recognition, parallel computing, associative memory, and combinatorial optimization. Such applications heavily depend on the dynamical behaviors. Thus, the qualitative analysis of the dynamical behaviors is a necessary step for the practical design and application of neural networks.

The stability of Cohen-Grossberg neural network with or without delays has been widely studied by many researchers, and various interesting result have

Received December 6, 2009; Revised September 29, 2010

2010 Mathematics Subject Classification. 34D23, 39A13.

Key words and phrases. periodic solutions, two-dimensional discrete-time CohenGrossberg BAM neural networks, global exponential stability, coincidence degree theory, Lyapunov functional.

This project was supported by Doctoral Fund of Ministry of Education of China (No:200805321017), Postdoctoral fund of China (No:20060400267), Fund of Nation Nature Science (No.61065008). 
been reported ([2]-[25]). As is well known, the studies on neural dynamical system not only involve a discussion of stability properties, but also involve other dynamic behavior, such as periodic oscillatory behavior, chaos and bifurcation. In many applications, the periodic oscillatory behavior is of great interest, it has been found applications in learning theory [39] which is motivated by the fact that learning usually requires repetition. Hence, it is of prime importance to study periodic oscillatory solutions of neural networks. Recent years, a few of authors discussed the existence and stability of periodic solution to twodimensional Cohen-Grossberg neural networks with delays [3, 37]. However, the existence and stability result of periodic solution to two-dimensional discrete Cohen-Grossberg neural networks with delays have been not found in the present literature. On the other hand, recent years, the stability of equilibrium point and periodic solutions for continuous-time and discrete time BAM neural networks has also be widely investigated and many interesting results have be obtained, for example, see [35]-[45].

Motivated by above works, in this paper, we are concerned with the existence and stability of periodic solution to two-dimensional discrete-time CohenGrossberg BAM neural networks with delays.

Recently, some authors studied dynamical behavior for discrete-time analogues of neural networks by semi-discrete method, see [21, 23, 22, 14, 30]. Just as said in [21], semi-discrete method has more advantages than Euler method in preserving the dynamics of continuous model. Different from those in neural networks models in $[21,23,22,14]$, in Cohen-Grossberg neural networks, the amplification function is nonlinear, which brings some difficulties in using semi-discrete method to discrete Cohen-Grossberg neural networks. In [7], by using semi-discrete method, a new discrete analogue of one-dimensional continuous Cohen-Grossberg neural networks with varying delays is obtained by analysis and approximation techniques.

In this paper, we consider discrete analogue of the following two-dimensional continuous Cohen-Grossberg BAM neural networks:

$$
\left\{\begin{array}{l}
\frac{d x_{i}(t)}{d t}=-a_{i}\left(t, x_{i}(t)\right)\left[b_{i}\left(t, x_{i}(t)\right)-\sum_{j=1}^{m} p_{i j}(t) f_{j}\left(y_{j}\left(t-\tau_{i j}(t)\right)\right)-I_{i}(t)\right] \\
\frac{d y_{j}(t)}{d t}=-c_{j}\left(t, y_{j}(t)\right)\left[d_{j}\left(t, y_{j}(t)\right)-\sum_{i=1}^{k} q_{j i}(t) g_{i}\left(x_{i}\left(t-\sigma_{j i}(t)\right)\right)-J_{j}(t)\right]
\end{array}\right.
$$

where $i=1,2, \ldots, k, j=1,2, \ldots, m, k, m \geq 2$ are the number of neurons in the networks; $x_{i}(t)$ and $y_{j}(t)$ are the states of the $i$ th neuron from the neural field $F_{X}$ and the $j$ th neuron from the neural field $F_{Y}$ at the time $t$, respectively; $f_{i}, g_{i}$ denote the activation functions of $j$ th neuron from $F_{Y}$ and the $i$ th neuron from $F_{X}$, respectively; $p_{i j}(t)$ weights the strength of the $i$ th neuron on the $j$ th neuron at the time $t ; q_{j i}(t)$ weights the strength of the $j$ th neuron on the $i$ th neuron at the time $t ; I_{i}(t), J_{j}(t)$ denote the external inputs on the $i$ th neuron from $F_{X}$ and the $j$ th neuron from $F_{Y}$, respectively; $a_{i}\left(t, x_{i}(t)\right)$ and $d_{j}\left(t, y_{j}(t)\right)$ are 
appropriately behaved functions such that the solution of model (1.1) remain bounded.

Assume that

$\left(H_{1}\right) a_{i}(t, x), b_{i}(t, x), c_{j}(t, y), d_{j}(t, y): \mathbb{R} \times \mathbb{R} \rightarrow \mathbb{R}$ are continuous $\omega$-periodic functions with $\omega>0$ with respect to the first variable for $\forall t, x, y \in \mathbb{R}, i=$ $1, \ldots, k, j=1, \ldots, m, \tau_{i j}(t)>0, \sigma_{j i}(t)>0, p_{i j}(t), q_{j i}(t), I_{i}(t), J_{j}(t): \mathbb{R} \times \mathbb{R} \rightarrow$ $\mathbb{R}$ are continuous $\omega$-periodic functions for $t \in \mathbb{R}, i=1, \ldots, k, j=1, \ldots, m$;

$\left(H_{2}\right)$ there exist positive continuous $\omega$-periodic functions $\gamma_{i}, \gamma_{j}^{*}, \Gamma_{i}, \Gamma_{j}^{*}$ such that for $\forall t, x, y \in \mathbb{R}, i=1, \ldots, k, j=1, \ldots, m$,

$$
\begin{gathered}
0<\gamma_{i}(t) \leq \frac{a_{i}(t, x) b_{i}(t, x)-a_{i}(t, y) b_{i}(t, y)}{x-y}<\Gamma_{i}(t) \\
0<\gamma_{j}^{*}(t) \leq \frac{c_{j}(t, x) d_{j}(t, x)-c_{j}(t, y) d_{j}(t, y)}{x-y}<\Gamma_{j}^{*}(t)
\end{gathered}
$$

$\left(H_{3}\right)$ there exist positive constants $\overline{a_{i}}, \overline{c_{j}}$ such that for $\forall t, x, y \in \mathbb{R}, i=1, \ldots, k$, $j=1, \ldots, m$,

$$
0 \leq a_{i}(t, x) \leq \overline{a_{i}}, 0 \leq c_{j}(t, y)<\overline{c_{j}} .
$$

Using the same semi-discrete method as that in [7], we can obtain the discrete analogue of continuous two-dimensional Cohen-Grossberg BAM neural networks (1.1) as follows:

$$
\left\{\begin{aligned}
& x_{i}(n+1) \\
= & x_{i}(n) e^{-\frac{\Gamma_{i}(n)+\gamma_{i}(n)}{2} h}+\xi_{i}(h)\left\{\frac{\Gamma_{i}(n)+\gamma_{i}(n)}{2} x_{i}(n)\right. \\
& \left.-a_{i}\left(n, x_{i}(n)\right)\left[b_{i}\left(n, x_{i}(n)\right)-\sum_{j=1}^{m} p_{i j}(n) f_{j}\left(y_{j}\left(n-\tau_{i j}(n)\right)\right)+I_{i}(n)\right]\right\} \\
& y_{j}(n+1) \\
= & y_{j}(n) e^{-\frac{\Gamma_{j}^{*}(n)+\gamma_{j}^{*}(n)}{2} h}+\eta_{j}(h)\left\{\frac{\Gamma_{j}^{*}(n)+\gamma_{j}^{*}(n)}{2} y_{j}(n)\right. \\
& \left.-c_{j}\left(n, y_{j}(n)\right)\left[d_{j}\left(n, y_{j}(n)\right)-\sum_{i=1}^{k} q_{j i}(n) g_{i}\left(x_{i}\left(n-\sigma_{j i}(n)\right)\right)+J_{j}(n)\right]\right\}
\end{aligned}\right.
$$

where $\xi_{i}(h)=\frac{1-e^{-\frac{\Gamma_{i}(n)+\gamma_{i}(n)}{2} h}}{\frac{\Gamma_{i}(n)+\gamma_{i}(n)}{2}}=h+O\left(h^{2}\right), i=1, \ldots, k, k \in \mathbb{Z}_{0}^{+} ; \eta_{j}(h)=$ $\frac{1-e^{-\frac{\Gamma_{j}^{*}(n)+\gamma_{j}^{*}(n)}{2}} h}{\frac{\Gamma_{j}^{*}(n)+\gamma_{j}^{*}(n)}{2}}=h+O\left(h^{2}\right), j=1, \ldots, m, m \in \mathbb{Z}_{0}^{+}, \mathbb{Z}_{0}^{+}$will be defined below.

Since the existence and global exponential stability of periodic solution of system (1.2) have been not investigated, so in this paper, our propose is to obtain sufficient condition to guarantee the existence and global exponential stability of periodic solution for system (1.2). The paper is organized as follows. In Section 2, the existence of periodic solution for discrete Cohen-Grossberg BAM system (1.2) is studied by using continuation theorem of coincidence 
degree theory. In Section 3, the global exponential stability of periodic solution is established by constructing a Lyapunov functional. In Section 4, an example is given to show the effectiveness of the results in this paper.

Compared with the results obtained in [7], [43], [44], in our result on the existence of periodic solution, the boundedness conditions on the activation functions in [7], [43] and [44] are replaced with global Lipschitz conditions. Compared with the result obtained in [4], in our result on the existence and global exponential stability of periodic solution, the assumptions that the value of activation functions at zero is zero are removed. Therefore, we obtain new sufficient conditions on the existence and global exponential stability of periodic solution for discrete-time neural networks.

Let $\mathbb{Z}$ be the set of all integers, i.e., $\mathbb{Z}=\{\ldots,-2,-1,0,1,2, \ldots\} ; \mathbb{Z}_{0}^{+}=$ $\{0,1,2, \ldots\} ;[a, b]_{\mathbb{Z}}=\{a, a+1, \ldots, b-1, b\}$ where $a, b \in \mathbb{Z}, a \leq b$. Especially, $[a, \infty)_{\mathbb{Z}} \stackrel{\text { def }}{=}\{a, a+1, a+2, \ldots\},(-\infty, 0]_{\mathbb{Z}} \stackrel{\text { def }}{=}\{\ldots,-2,-1,0\}$, where $a \in \mathbb{Z}$. It is not difficult to verify that $\xi_{i}(h)>0, \eta_{j}(h)>0$. In studying the discrete-time analogue (2), we assume that $h \in(0,+\infty), a_{i}(n, x), c_{j}(n, y)$ : $\mathbb{Z} \times \mathbb{R} \rightarrow[0,+\infty], \tau_{i j}(n), \sigma_{j i}(n): \mathbb{Z} \rightarrow \mathbb{Z}_{0}^{+}, b_{i}(n, x), d_{j}(n, y): \mathbb{Z} \times \mathbb{R} \rightarrow \mathbb{R}$, $p_{i j}(n), q_{j i}(n), I_{i}(n), J_{j}(n): \mathbb{Z} \rightarrow \mathbb{R}$ for $i=1,2, \ldots, k, j=1, \ldots, m$.

System (1.2) is supplemented with initial values of the form given by $x_{i}(l)=$ $\phi_{i}(l), y_{j}(l)=\psi_{j}(l)$ for $i=1,2, \ldots, k, j=1, \ldots, m, l \in(-\infty, 0]_{\mathbb{Z}}$, where $(-\infty, 0]_{\mathbb{Z}}=\{\ldots,-2,-1,0\}, \phi_{i}(l)$ and $\psi_{j}(l)$ defined for $l \in(-\infty, 0]_{\mathbb{Z}}$ denote sequences of real numbers and $\sup _{l \in(-\infty, 0]_{\mathbb{Z}}}\left|\phi_{i}(l)\right|<\infty, \sup _{l \in(-\infty, 0]_{\mathbb{Z}}}\left|\psi_{j}(l)\right|<\infty$, $i=1, \ldots, k, j=1, \ldots, m$.

\section{Existence of at least a periodic solution}

In this paper, based on the Mawhin continuation theorem, we will study the existence of periodic solution of the system (1.2). For convenience, we first introduce the Mawhin continuity theorem proposed by Gaines and Mawhin $[12]$.

Let $X, Y$ be Banach spaces, $L$ : Dom $L \subset X \rightarrow Y$ be a linear mapping and $N: X \rightarrow Y$ be a continuous mapping. The mapping $L$ will be called a Fredholm mapping of index zero if $\operatorname{dim} \operatorname{Ker} L=\operatorname{codim} \operatorname{Im} L<\infty$ and $\operatorname{Im} L$ is closed in $Y$. If $L$ is a Fredholm mapping of index zero, then there exist continuous projectors $P: X \rightarrow X$ and $Q: Y \rightarrow Y$ such that $\operatorname{Im} P=\operatorname{Ker} L$ and $\operatorname{Im} L=\operatorname{Ker} Q=\operatorname{Im}(I-Q)$. It follows that $L / \operatorname{Dom} L \cap \operatorname{Ker} P:(I-P) X \rightarrow \operatorname{Im} L$ is invertible. We denote the inverse of the map $L / \operatorname{Dom} L \cap \operatorname{Ker} P$ by $K_{p}$. If $\Omega$ is an open bounded subset of $X$, the mapping $N$ will be called $L$-compact on $\bar{\Omega}$ if $(Q N)(\bar{\Omega})$ is bounded and $K_{p}(I-Q) N: \bar{\Omega} \rightarrow X$ is compact. Since $\operatorname{Im} Q$ is isomorphic to $\operatorname{Ker} L$, there exists an isomorphism $J: \operatorname{Im} Q \rightarrow \operatorname{Ker} L$.

In the proof of our existence theorem, we will use the continuation theorem of Gaines and Mawhin ([12]).

Lemma 2.1 (Continuation Theorem). Let L be a Fredholm mapping of index zero and let $N$ be $L$-compact on $\bar{\Omega}$. Suppose 
(i) $L x \neq \lambda N(x), \forall \lambda \in(0,1), x \in \partial \Omega$;

(ii) $Q N x \neq 0, \forall x \in \operatorname{Ker} L \cap \partial \Omega$;

(iii) $\operatorname{deg}(J Q N x, \Omega \cap \operatorname{Ker} L, 0) \neq 0$.

Then $L x=N x$ has at least one solution in Dom $L \cap \bar{\Omega}$.

Let $|\cdot|$ denotes the norm in $\mathbb{R}$, for the sake of convenience, we introduce some notations.

$$
\begin{aligned}
& I_{\omega}=\{0,1, \ldots, \omega-1\}, b_{i}^{*}=\max _{n \in I_{\omega}}\left|b_{i}(n, 0)\right|, d_{j}^{*}=\max _{n \in I_{\omega}}\left|d_{j}(n, 0)\right|, \\
& \left.\underline{f}=\min _{n \in I_{\omega}}\{|f(n)|\}, \bar{f}=\max _{n \in I_{\omega}}|f(n)|\right\},
\end{aligned}
$$

where $f(n)$ is a real number sequence. Throughout this paper, for system (1.2), in addition to $\left(H_{2}\right)$ and $\left(H_{3}\right)$, we further suppose that

$\left(H_{4}\right)$ There exist nonnegative constants $\alpha_{j}, \beta_{i}(i=1, \ldots, k, j=1,2, \ldots, m)$ such that for $\forall x, y \in \mathbb{R}$

$$
\begin{gathered}
\left|f_{j}(x)-f_{j}(y)\right| \leq \alpha_{j}|x-y|, \\
\left|g_{i}(x)-g_{i}(y)\right| \leq \beta_{i}|x-y|
\end{gathered}
$$

$\left(H_{5}\right)$ There exist positive constants $M_{j}, N_{i}(i=1, \ldots, k, j=1, \ldots, m)$ such that

$$
\left|f_{j}(x)\right| \leq M_{j}, \quad\left|g_{i}(x)\right| \leq N_{i}
$$

$\left(H_{6}\right) a_{i}\left(n, x_{i}\right), c_{j}\left(n, y_{j}\right), b_{i}\left(n, x_{i}\right), d_{j}\left(n, y_{j}\right)$ are continuous $\omega$-periodic functions with respect to the first variable, $p_{i j}, q_{j i}, I_{i}, J_{i}$ are all $\omega$-periodic functions, where $i=1, \ldots, k, j=1,2, \ldots, m, \omega$ is a positive integer.

$\left(H_{7}\right)$ There exist positive continuous $\omega$-periodic functions $L_{i}^{a}(t), L_{j}^{c}(t)$ such that for $\forall x, y, t \in \mathbb{R}, i=1, \ldots, k, j=1, \ldots, m$,

$$
\begin{aligned}
& \left|a_{i}(t, x)-a_{i}(t, y)\right| \leq L_{i}^{a}(t)|x-y|, \\
& \left|c_{j}(t, x)-c_{j}(t, y)\right| \leq L_{j}^{c}(t)|x-y| .
\end{aligned}
$$

Denote $z(n)=\left(z_{1}(n), \ldots, z_{m+k}(n)\right)^{T}=\left(x_{1}(n), \ldots, x_{k}(n), y_{1}(n), \ldots, y_{m}(n)\right)^{T}$. Let $g_{m}=\left\{z=\{z(n)\}: z(n) \in \mathbb{R}^{m+k}, n \in \mathbb{Z}\right\}$, and $g^{\omega} \subset g_{m}$ be the subspace of all $\omega$ periodic sequence equipped with the norm $\|\cdot\|$, i.e., $\|z\|=$ $\sum_{i=1}^{k}\left(\max _{n \in I_{\omega}}\left|x_{i}(n)\right|\right)+\sum_{j=1}^{m}\left(\max _{n \in I_{\omega}}\left|y_{j}(n)\right|\right)$ for any $z(n) \in g^{\omega}$. It is easy to show that $g^{\omega}$ is a finite dimensional Banach space. Let $g_{0}^{\omega}=\{z=\{z(n)\} \in$ $\left.g^{\omega}: \sum_{n=0}^{\omega-1} z(n)=0\right\}, g_{c}^{\omega}=\left\{z=\{z(n)\} \in g^{\omega}: z(n)=c \in \mathbb{R}^{m+k}, n \in \mathbb{Z}\right\}$, then it is easy to check that $g_{0}^{\omega}$ and $g_{c}^{\omega}$ are both chosen linear subspace of $g^{\omega}$ and $g^{\omega}=g^{\omega} \oplus g_{c}^{\omega}, \operatorname{dim} g_{c}^{\omega}=m+k$.

Our main result on the existence of at least a periodic solutions for the system (1.2) is stated in the following theorem.

Theorem 2.1. Assume that $\left(H_{2}\right)-\left(H_{4}\right)$ and $\left(H_{6}\right)$ hold. Further assume that $\left(h_{1}\right)$ For $i=1,2, k, j=1, \ldots, m$,

$$
\underline{\gamma_{i}}>\overline{a_{i}} \sum_{j=1}^{m} \overline{p_{i j}} \alpha_{j}
$$


and

$$
\underline{\gamma_{j}^{*}}>\overline{c_{j}} \sum_{i=1}^{k} \overline{q_{j i}} \beta_{i} .
$$

Then the system (1.2) has at least one $\omega$-periodic solution.

Proof. In order to apply Lemma 2.1 to the system (1.2). Let $X=Y=g^{\omega}$ and define

for $z \in X, n \in \mathbb{Z}$, where

$$
N z(n)=\left[\begin{array}{c}
F_{1}(n) \\
\ldots \ldots \ldots \ldots \\
F_{k}(n) \\
\ldots \ldots \ldots \ldots \\
G_{1}(n) \\
\ldots \ldots \ldots \ldots \\
G_{m}(n)
\end{array}\right]
$$

$$
\begin{aligned}
F_{1}(n)= & \left(e^{-\frac{\Gamma_{1}(n)+\gamma_{1}(n)}{2} h}-1\right) x_{1}(n)+\xi_{1}(h)\left\{\frac{\Gamma_{1}(n)+\gamma_{1}(n)}{2} x_{1}(n)-a_{1}\left(n, x_{1}(n)\right)\right. \\
& \left.\times\left[b_{1}\left(n, x_{1}(n)\right)-\sum_{j=1}^{m} p_{1 j}(n) f_{j}\left(y_{j}\left(n-\tau_{1 j}(n)\right)\right)+I_{1}(n)\right]\right\}, \\
\ldots \ldots \ldots \ldots & \ldots \\
F_{k}(n)= & \left(e^{-\frac{\Gamma_{k}(n)+\gamma_{k}(n)}{2} h}-1\right) x_{k}(n)+\xi_{k}(h)\left\{\frac{\Gamma_{k}(n)+\gamma_{k}(n)}{2} x_{k}(n)-a_{k}\left(n, x_{k}(n)\right)\right. \\
& \left.\times\left[b_{k}\left(n, x_{k}(n)\right)-\sum_{j=1}^{m} p_{k j}(n) f_{j}\left(y_{j}\left(n-\tau_{k j}(n)\right)\right)+I_{k}(n)\right]\right\}, \\
G_{1}(n)=( & \left.e^{-\frac{\Gamma_{1}^{*}(n)+\gamma_{1}^{*}(n)}{2} h}-1\right) y_{1}(n)+\eta_{1}(h)\left\{\frac{\Gamma_{1}^{*}(n)+\gamma_{1}^{*}(n)}{2} y_{1}(n)\right. \\
& \left.-c_{1}\left(n, y_{1}(n)\right)\left[d_{1}\left(n, y_{1}(n)\right)-\sum_{i=1}^{m} q_{i 1}(n) g_{i}\left(x_{i}\left(n-\sigma_{1 i}(n)\right)\right)+J_{1}(n)\right]\right\}, \\
& \left.\times\left[d_{m}\left(n, y_{m}(n)\right)-\sum_{i=1}^{m} q_{i m}(n) g_{i}\left(x_{i}\left(n-\sigma_{m i}(n)\right)\right)+J_{m}(n)\right]\right\} . \\
G_{m}(n)= & \left(e^{-\frac{\Gamma_{m}^{*}(n)+\gamma_{m}^{*}(n)}{2} h}-1\right) y_{m}(n)+\eta_{m}(h)\left\{\frac{\Gamma_{m}^{*}(n)+\gamma_{m}^{*}(n)}{2} y_{m}(n)-c_{m}\left(n, y_{m}(n)\right)\right. \\
&
\end{aligned}
$$

Let $L z(n)=z(n+1)-z(n), P z=Q z=\frac{1}{\omega} \sum_{s=0}^{\omega-1} z(s), z \in X($ or $Y), n, s \in \mathbb{Z}$. It is easy to see that $L$ is a bounded linear operator. $\operatorname{Ker} L=g_{c}^{\omega}, \operatorname{Im} L=g_{0}^{\omega}$ is closed in $Y$, and $\operatorname{dim} \operatorname{Ker} L=m+k=\operatorname{codim} \operatorname{Im} L$. Clearly, $P$ and $Q$ are continuous projectors such that $\operatorname{Im} P=\operatorname{Ker} L, \operatorname{lm} L=\operatorname{Ker} Q=\operatorname{Im}(I-Q)$. Thus, there exists $K_{p}: \operatorname{Im} L \rightarrow \operatorname{Ker} P \cap \operatorname{Dom} L$, which is the converse projectors of $L$, and $K_{p}(n)=\sum_{r=0}^{n-1} z(r)-\frac{1}{\bar{\omega}} \sum_{r=1}^{\omega} \sum_{s=0}^{r-1} z(s), n \in[0, \omega-1]$. 
Employing Lebesgue's convergence theorem, we can easily prove that $Q N$ and $K_{p}(I-Q) N$ are continuous. By Ascoli-Arzela's theorem, we can obtain that $Q N(\bar{\Omega})$ and $K_{p}(I-Q) N$ are relatively compact for any open bounded set $\Omega \in X$. Hence, $N$ is $L$-compact on $\bar{\Omega}$.

Corresponding to operator equation $L z=\lambda N z, \lambda \in(0,1)$, we have for $i=1,2, \ldots, k, j=1 \ldots, m$,

$$
\left\{\begin{array}{l}
x_{i}(n+1)-x_{i}(n)=\lambda F_{i}(n), \\
y_{i}(n+1)-y_{i}(n)=\lambda G_{j}(n) .
\end{array}\right.
$$

Suppose that $z(n)=\left(x_{1}(n), \ldots, x_{k}(n), y_{1}(n), \ldots, y_{m}(n)\right)^{T} \in X$ is a solution of system (2.1) for a certain $\lambda \in(0,1)$. Condition $\left(H_{4}\right)$ implies that for $\forall x, y \in \mathbb{R}$, $i=1,2, \ldots, k, j=1,2, \ldots, m$,

$$
\left|f_{j}(x)\right| \leq\left|f_{j}(0)\right|+\alpha_{j}|x|
$$

and

$$
\left|g_{i}(y)\right| \leq\left|g_{i}(0)\right|+\beta_{i}|y|
$$

In view of system (2.1), inequalities (2.2) and (2.3) we have

$$
\begin{aligned}
& \max _{n \in I_{\omega}}\left|x_{i}(n)\right| \\
= & \max _{n \in I_{\omega}}\left|x_{i}(n+1)\right| \\
\leq & \max _{n \in I_{\omega}}\left\{\left(1+\lambda\left(e^{-\frac{\Gamma_{i}(n)+\gamma_{i}(n)}{2} h}-1\right)\right)\left|x_{i}(n)\right|+\lambda \xi_{i}(h)\left[\mid \frac{\Gamma_{i}(n)+\gamma_{i}(n)}{2} x_{i}(n)\right.\right. \\
& \left.\left.-a_{i}\left(n, x_{i}(n)\right)\left(b_{i}\left(n, x_{i}(n)\right)-\sum_{j=1}^{m} p_{i j}(n) f_{j}\left(y_{j}\left(n-\tau_{i j}(n)\right)\right)+I_{i}(n)\right) \mid\right]\right\} \\
\leq & \left(1+\lambda\left(e^{-\frac{\Gamma_{i}(n)+\gamma_{i}(n)}{2} h}-1\right) \frac{2 \underline{\gamma_{i}}}{\Gamma_{i}(n)+\gamma_{i}(n)}\right) \times \max _{n \in I_{\omega}}\left|x_{i}(n)\right| \\
& +\lambda \xi_{i}(h)\left\{\overline{a_{i}}\left[b_{i}^{*}+\sum_{j=1}^{m} \overline{p_{i j}}\left[\left|f_{j}(0)\right|+\alpha_{j} \max _{n \in I_{\omega}}\left|y_{j}(n)\right|\right]+\overline{I_{i}}\right]\right\}
\end{aligned}
$$

and

$$
\text { (2.5) } \begin{aligned}
& \max _{n \in I_{\omega}}\left|y_{j}(n)\right| \\
= & \max _{n \in I_{\omega}}\left|y_{j}(n+1)\right| \\
\leq & \max _{n \in I_{\omega}}\left\{\left(1+\lambda\left(e^{-\frac{\Gamma_{j}^{*}(n)+\gamma_{j}^{*}(n)}{2} h}-1\right)\right)\left|y_{j}(n)\right|\right. \\
& +\lambda \eta_{j}(h)\left[\mid \frac{\Gamma_{j}(n)+\gamma_{j}(n)}{2} y_{j}(n)-c_{j}\left(n, y_{j}(n)\right)\left(d_{j}\left(n, y_{j}(n)\right)-\sum_{i=1}^{k} q_{j i}(n)\right.\right. \\
& \left.\left.\left.\times g_{i}\left(x_{i}\left(n-\sigma_{j i}(n)\right)\right)+J_{j}(n)\right) \mid\right]\right\}
\end{aligned}
$$




$$
\begin{aligned}
\leq & \left(1+\lambda\left(e^{-\frac{\Gamma_{j}^{*}(n)+\gamma_{j}^{*}(n)}{2} h}-1\right) \frac{2 \gamma_{j}^{*}(n)}{\Gamma_{j}^{*}(n)+\gamma_{j}^{*}(n)}\right) \max _{n \in I_{\omega}}\left|y_{j}(n)\right| \\
& +\lambda \eta_{j}(h)\left\{\overline{c_{j}}\left[d_{j}^{*}+\sum_{i=1}^{k} \overline{q_{j i}}\left[\left|g_{i}(0)\right|+\beta_{i} \max _{n \in I_{\omega}}\left|x_{i}(n)\right|\right]+\overline{J_{j}}\right]\right\} .
\end{aligned}
$$

Setting

$$
\left.\left.\left.\left.\max _{1 \leq j \leq m}\left\{\max _{n \in I_{\omega}}\left|y_{j}(n)\right|\right)\right\}=\max _{n \in I_{\omega}}\left|y_{j_{0}}(n)\right|\right), \max _{1 \leq i \leq k}\left\{\max _{n \in I_{\omega}}\left|x_{i}(n)\right|\right)\right\}=\max _{n \in I_{\omega}}\left|x_{i_{0}}(n)\right|\right),
$$

where $j_{0} \in\{1,2, \ldots, m\}, i_{0} \in\{1,2, \ldots, k\}$. Then from (2.4) and (2.5), we have

$$
\begin{aligned}
& \max _{n \in I_{\omega}}\left|x_{i_{0}}(n)\right| \\
\leq & \left(1+\lambda\left(e^{-\frac{\Gamma_{i}(n)+\gamma_{i}(n)}{2} h}-1\right) \frac{2 \underline{\gamma_{i}}}{\Gamma_{i}(n)+\gamma_{i}(n)}\right) \max _{n \in I_{\omega}}\left|x_{i_{0}}(n)\right| \\
& +\lambda \xi_{i}(h)\left\{\overline{a_{i}}\left[b_{i}^{*}+\sum_{j=1}^{m} \overline{p_{i j}}\left[\left|f_{j}(0)\right|+\alpha_{j} \max _{n \in I_{\omega}}\left|y_{j_{0}}(n)\right|\right]+\overline{I_{i}}\right]\right\}
\end{aligned}
$$

and

$$
\begin{aligned}
& \max _{n \in I_{\omega}}\left|y_{j_{0}}(n)\right| \\
\leq & \left(1+\lambda\left(e^{-\frac{\Gamma_{j}^{*}(n)+\gamma_{j}^{*}(n)}{2} h}-1\right) \frac{2 \gamma_{j}^{*}(n)}{\Gamma_{j}^{*}(n)+\gamma_{j}^{*}(n)}\right) \max _{n \in I_{\omega}}\left|y_{j_{0}}(n)\right| \\
& +\lambda \eta_{j}(h)\left\{\overline{c_{j}}\left[d_{j}^{*}+\sum_{i=1}^{k} \overline{q_{j i}}\left[\left|g_{i}(0)\right|+\beta_{i} \max _{n \in I_{\omega}}\left|x_{i_{0}}(n)\right|\right]+\overline{J_{j}}\right]\right\} .
\end{aligned}
$$

That is

$$
\begin{aligned}
& \left(1-e^{-\frac{\Gamma_{i}(n)+\gamma_{i}(n)}{2} h}\right) \frac{2 \underline{\gamma_{i}}}{\Gamma_{i}(n)+\gamma_{i}(n)} \max _{n \in I_{\omega}}\left|x_{i_{0}}(n)\right| \\
\leq & \frac{1-e^{-\frac{\Gamma_{i}(n)+\gamma_{i}(n)}{2} h}}{\frac{\Gamma_{i}(n)+\gamma_{i}(n)}{2}}\left\{\overline{a_{i}}\left[b_{i}^{*}+\sum_{j=1}^{m} \overline{p_{i j}}\left[\left|f_{j}(0)\right|+\alpha_{j} \max _{n \in I_{\omega}}\left|y_{j_{0}}(n)\right|\right]+\overline{I_{i}}\right]\right\}
\end{aligned}
$$

and

$$
\begin{aligned}
& \left(1-e^{-\frac{\Gamma_{i}^{*}(n)+\gamma_{i}^{*}(n)}{2} h}\right) \frac{2 \gamma_{i}^{*}}{\Gamma_{i}^{*}(n)+\gamma_{i}^{*}(n)} \max _{n \in I_{\omega}}\left|y_{j_{0}}(n)\right| \\
\leq & \frac{1-e^{-\frac{\Gamma_{j}^{*}(n)+\gamma_{j}^{*}(n)}{2} h}}{\frac{\Gamma_{j}^{*}(n)+\gamma_{j}^{*}(n)}{2}}\left\{\overline{c_{j}}\left[d_{j}^{*}+\sum_{i=1}^{k} \overline{q_{j i}}\left[\left|g_{i}(0)\right|+\beta_{i} \max _{n \in I_{\omega}}\left|x_{i_{0}}(n)\right|\right]+\overline{J_{j}}\right]\right\} .
\end{aligned}
$$

From (2.6) and (2.7), we obtain

(2.8) $\underline{\gamma}_{i} \max _{n \in I_{\omega}}\left|x_{i_{0}}(n)\right| \leq \overline{a_{i}}\left[b_{i}^{*}+\overline{I_{i}}+\sum_{j=1}^{m} \overline{p_{i j}}\left|f_{j}(0)\right|\right]+\overline{a_{i}} \sum_{j=1}^{m} \overline{p_{i j}} \alpha_{j} \max _{I \in I_{\omega}}\left|y_{j_{0}}(n)\right|$ 
and

(2.9) $\underline{\gamma_{j}^{*}} \max _{n \in I_{\omega}}\left|y_{j_{0}}(n)\right| \leq \overline{c_{j}}\left[d_{j}^{*}+\overline{J_{j}}+\sum_{i=1}^{k} \overline{q_{j i}}\left|g_{i}(0)\right|\right]+\overline{c_{j}} \sum_{i=1}^{k} \overline{q_{j i}} \beta_{i} \max _{I \in I_{\omega}}\left|x_{i_{0}}(n)\right|$.

We consider two possible cases:

(i) $\max _{n \in I_{\omega}}\left|y_{j_{0}}(n)\right| \leq \max _{n \in I_{\omega}}\left|x_{i_{0}}(n)\right|$;

(ii) $\max _{n \in I_{\omega}}\left|y_{j_{0}}(n)\right|>\max _{n \in I_{\omega}}\left|x_{i_{0}}(n)\right|$.

(i) When $\max _{n \in I_{\omega}}\left|y_{j_{0}}(n)\right| \leq \max _{n \in I_{\omega}}\left|x_{i_{0}}(n)\right|$, from (2.8), we have

$$
\left(\underline{\gamma_{i}}-\overline{a_{i}} \sum_{j=1}^{m} \overline{p_{i j}} \alpha_{j}\right) \max _{n \in I_{\omega}}\left|x_{i_{0}}(n)\right| \leq \overline{a_{i}}\left(b_{i}^{*}+\overline{I_{i}}+\sum_{j=1}^{m} \overline{p_{i j}}\left|f_{j}(0)\right|\right) .
$$

Then

$$
\begin{aligned}
\max _{n \in I_{\omega}}\left|y_{j_{0}}(n)\right| & \leq \max _{n \in I_{\omega}}\left|x_{i_{0}}(n)\right| \\
& \leq \max _{1 \leq i \leq k}\left\{\frac{\overline{a_{i}}\left(b_{i}^{*}+\overline{I_{i}}+\sum_{j=1}^{m} \overline{p_{i j}}\left|f_{j}(0)\right|\right)}{\underline{\gamma_{i}}-\overline{a_{i}} \sum_{j=1}^{m} \overline{p_{i j}} \alpha_{j}}\right\} \stackrel{\text { def }}{=} d_{1} .
\end{aligned}
$$

(ii) When $\max _{n \in I_{\omega}}\left|y_{j_{0}}(n)\right|>\max _{n \in I_{\omega}}\left|x_{i_{0}}(n)\right|$, from (2.9), we have

$$
\left.\underline{\left(\gamma_{i}^{*}\right.}-\overline{c_{j}} \sum_{i=1}^{k} \overline{q_{j i}} \beta_{i}\right) \max _{n \in I_{\omega}}\left|y_{j_{0}}(n)\right| \leq \overline{c_{j}}\left(d_{j}^{*}+\overline{J_{j}}+\sum_{i=1}^{k} \overline{q_{j i}}\left|g_{i}(0)\right|\right) .
$$

Then

$$
\begin{aligned}
\max _{n \in I_{\omega}}\left|x_{i_{0}}(n)\right| & <\max _{n \in I_{\omega}}\left|y_{j_{0}}(n)\right| \\
& \leq \max _{1 \leq j \leq m}\left\{\frac{\overline{c_{j}}\left(d_{j}^{*}+\overline{J_{j}}+\sum_{i=1}^{k} \overline{q_{j i}}\left|g_{i}(0)\right|\right)}{\underline{\gamma_{j}^{*}}-\overline{c_{j}} \sum_{i=1}^{k} \overline{q_{j i}} \beta_{i}}\right\} \stackrel{\text { def }}{=} d_{2} .
\end{aligned}
$$

From (2.11) and (2.12), we have for $n \in I_{\omega}, i=1,2, \ldots, k, j=1, \ldots, m$,

$$
\left|x_{i}(n)\right|<\max \left\{d_{1}, d_{2}\right\} \stackrel{\text { def }}{=} d
$$

and

$$
\left|y_{j}(n)\right|<\max \left\{d_{1}, d_{2}\right\} \stackrel{\text { def }}{=} d \text {. }
$$

Obviously, $d_{1}, d_{2}, d$ are independent of $\lambda$. Taking $\Omega=\{z \in X:\|z\|<k(d+$ $\left.\left.r_{1}\right)+m\left(d+r_{2}\right)\right\}$, where $r_{1}, r_{2}$ are two chosen positive constants such that the bound of $\Omega$ is larger. This $\Omega$ satisfies condition (i) in Lemma 2.1. Now, we 
prove that when $z \in \partial \Omega \cap \operatorname{Ker} L=\partial \Omega \cap \mathbb{R}^{m+k}, Q N z \neq 0$. If this is not true, then constant vector $z$ with $z \in \partial \Omega \cap \mathbb{R}^{m+k}$ such that for $i=1,2, \ldots, k, j=1, \ldots, m$

$$
\begin{aligned}
& \frac{1}{\omega} \sum_{s=0}^{\omega-1}\left\{\left(e^{-\frac{\Gamma_{i}(s)+\gamma_{i}(s)}{2} h}-1\right) x_{i}+\xi_{i}(h)\left\{\frac{\Gamma_{i}(s)+\gamma_{i}(s)}{2} x_{i}\right.\right. \\
& \left.-a_{i}\left(s, x_{i}\right)\left[b_{i}\left(s, x_{i}\right)-\sum_{j=1}^{m} p_{i j}(s) f_{j}\left(y_{j}\right)+I_{i}(s)\right]\right\}=0 \\
& \frac{1}{\omega} \sum_{s=0}^{\omega-1}\left\{\left(e^{-\frac{\Gamma_{j}^{*}(s)+\gamma_{j}^{*}(s)}{2} h}-1\right) y_{j}+\eta_{j}(h)\left\{\frac{\Gamma_{j}^{*}(s)+\gamma_{j}^{*}(s)}{2} y_{j}\right.\right. \\
& \left.-c_{j}\left(s, y_{j}\right)\left[d_{j}\left(s, y_{j}\right)-\sum_{i=1}^{k} q_{j i}(s) g_{i}\left(x_{i}\right)+J_{j}(s)\right]\right\}=0 .
\end{aligned}
$$

From (2.15) and (2.16). following the argument of (2.13) and (2.14) gives that for $i=1,2, \ldots, k, j=1, \ldots, m$

$$
\left|x_{i}\right|<d,\left|y_{j}\right|<d
$$

That is

$$
\|z\|<k\left(d+r_{1}\right)+m\left(d+r_{2}\right) .
$$

This contradicts $z \in \partial \Omega$. This proof of (ii) in Lemma 2.1 is complete. Finally, we prove that (iii) in Lemma 2.1 holds. We only need to prove that $\operatorname{deg}\left\{-J Q N z, \Omega \cap \operatorname{Ker} L,(0,0, \ldots, 0)^{T}\right\} \neq(0,0, \ldots, 0)^{T}$. Now we show that

$$
\begin{aligned}
& \operatorname{deg}\left\{-J Q N z, \Omega \cap \operatorname{Ker} L,(0,0, \ldots, 0)^{T}\right\} \\
= & \operatorname{deg}\left\{\left(p_{1} x_{1}, \ldots, q_{m} y_{m}\right)^{T}, \Omega \cap \operatorname{Ker} L,(0,0, \ldots, 0)^{T}\right\},
\end{aligned}
$$

where

$$
\begin{gathered}
p_{i}=\frac{1}{\omega} \sum_{s=0}^{\omega-1}\left(1-e^{-\frac{\Gamma_{i}(s)+\gamma_{i}(s)}{2} h}\right), q_{j}=\frac{1}{\omega} \sum_{s=0}^{\omega-1}\left(1-e^{-\frac{\Gamma_{j}^{*}(s)+\gamma_{j}^{*}(s)}{2} h}\right), \\
i=1, \ldots, k, j=1, \ldots, m .
\end{gathered}
$$

To this end, we define a mapping $\phi: \operatorname{Dom} L \times[0,1] \rightarrow X$ by

$$
\phi\left(x_{1}, x_{2}, \ldots, x_{k} ; y_{1}, \ldots, y_{m}, \mu\right)
$$




$$
=\left(\begin{array}{c}
-\frac{\mu}{\omega} \sum_{s=0}^{\omega-1} F_{1}(s)+(1-\mu) p_{1} x_{1} \\
\ldots \ldots \ldots \ldots \\
-\frac{\mu}{\omega} \sum_{s=0}^{\omega-1} F_{i}(s)+(1-\mu) p_{i} x_{i} \\
\ldots \ldots \ldots \\
-\frac{\mu}{\omega} \sum_{s=0}^{\omega-1} G_{1}(s)+(1-\mu) q_{1} y_{1} \\
\ldots \ldots \ldots \\
-\frac{\mu}{\omega} \sum_{s=0}^{\omega-1} G_{j}(s)+(1-\mu) q_{i}(s) y_{i} \\
\ldots \ldots \ldots
\end{array}\right)
$$

where $\mu \in[0,1]$ is a parameter. We show that when $z \in \partial \Omega \cap \operatorname{Ker} L=\partial \Omega \cap$ $\mathbb{R}^{m+k}, \phi\left(x_{1}, \ldots, x_{k}, y_{1}, \ldots, y_{m}, \mu\right) \neq(0, \ldots, 0)^{T}$. If it is not true, then when $z \in \partial \Omega \cap \operatorname{Ker} L=\partial \Omega \cap \mathbb{R}^{m+k}, \phi\left(x_{1}, x_{2}, \ldots, x_{k} ; y_{1}, \ldots, y_{m}, \mu\right)=(0,0, \ldots, 0)^{T}$. Thus constant vector $z$ with $z \in \partial \Omega$ satisfies for $i=1,2, \ldots, k, j=1, \ldots, m$,

$$
\begin{aligned}
& \frac{\mu}{\omega} \sum_{s=0}^{\omega-1}\left\{\left(1-e^{-\frac{\Gamma_{i}(s)+\gamma_{i}(s)}{2} h}\right) x_{i}-\xi_{i}(h)\left\{\frac{\Gamma_{i}(s)+\gamma_{i}(s)}{2} x_{i}\right.\right. \\
& \left.-a_{i}\left(s, x_{i}\right)\left[b_{i}\left(s, x_{i}\right)-\sum_{j=1}^{m} p_{i j}(s) f_{j}\left(y_{j}\right)+I_{i}(s)\right]\right\}+(1-\mu) p_{i} x_{i}=0, \\
& \frac{\mu}{\omega} \sum_{s=0}^{\omega-1}\left\{\left(1-e^{-\frac{\Gamma_{j}^{*}(s)+\gamma_{j}^{*}(s)}{2} h}\right) y_{j}-\eta_{j}(h)\left\{\frac{\Gamma_{j}^{*}(s)+\gamma_{j}^{*}(s)}{2} y_{j}\right.\right. \\
& \left.-c_{j}\left(s, y_{j}\right)\left[d_{j}\left(s, y_{j}\right)-\sum_{i=1}^{k} q_{j i}(s) g_{i}\left(x_{i}\right)+J_{j}(s)\right]\right\}+(1-\mu) q_{j} y_{j}=0 .
\end{aligned}
$$

Denote $\left|x_{i_{0}}\right|=\max _{1 \leq i \leq k}\left|x_{i}\right|,\left|y_{j_{0}}\right|=\max _{1 \leq j \leq m}\left|y_{j}\right|, i_{0} \in\{1, \ldots, k\}, j_{0}=$ $\{1, \ldots, m\}$. We make the following Claims.

Claim 1. $\left|x_{i_{0}}\right|<d+r_{1}$. Otherwise, $\left|x_{i_{0}}\right| \geq d+r_{1}$. We consider two possible cases:

(a) $\left|y_{j_{0}}\right| \leq\left|x_{i_{0}}\right|$

(b) $\left|y_{j_{0}}\right|>\left|x_{i_{0}}\right|$.

(a) When $\left|y_{i_{0}}\right| \leq\left|x_{i_{0}}\right|$, we have

$$
\begin{aligned}
& \frac{\mu}{\omega} \sum_{s=0}^{\omega-1}\left\{\left(1-e^{-\frac{\Gamma_{i}(s)+\gamma_{i}(s)}{2} h}\right) x_{i_{0}}-\xi_{i}(h)\left\{\frac{\Gamma_{i}(s)+\gamma_{i}(s)}{2} x_{i_{0}}\right.\right. \\
& \left.-a_{i}\left(s, x_{i_{0}}\right)\left[b_{i}\left(s, x_{i_{0}}\right)-\sum_{j=1}^{m} p_{i j} f_{j}\left(y_{j}\right)+I_{i}(n)\right]\right\}
\end{aligned}
$$




$$
\begin{aligned}
& +\frac{1-\mu}{\omega} \sum_{s=0}^{\omega-1}\left(1-e^{-\frac{\Gamma_{i}(s)+\gamma_{i}(s)}{2} h}\right) x_{i_{0}} \\
\geq & \frac{1}{\omega} \sum_{s=0}^{\omega-1}\left\{\left(1-e^{-\frac{\Gamma_{i}(s)+\gamma_{i}(s)}{2}}\right)\left|x_{i_{0}}\right|-\xi_{i}(h)\left[\frac{\Gamma_{i}(s)-\gamma_{i}(s)}{2}\left|x_{i_{0}}\right|\right.\right. \\
& \left.\left.-\overline{a_{i}}\left(\overline{b_{i}}+\sum_{j=1}^{m} \overline{p_{i j}}\left(\alpha_{j}\left|y_{j_{0}}\right|+\left|f_{j}(0)\right|\right)+\overline{I_{i}}\right)\right]\right\} \\
\geq & \frac{1}{\omega} \sum_{s=0}^{\omega-1}\left\{\gamma_{i}(s) \xi_{i}(h)\left|x_{i_{0}}\right|-\xi_{i}(h) \overline{a_{i}}\left[\overline{b_{i}}+\sum_{j=1}^{m} \overline{p_{i j}}\left(\alpha_{j}\left|x_{i_{0}}\right|+\left|f_{j}(0)\right|\right)+\overline{I_{i}}\right]\right\} \\
\geq & \frac{1}{\omega} \sum_{s=0}^{\omega-1}\left\{\underline{\gamma_{i}} \xi_{i}(h)\left|x_{i_{0}}\right|-\xi_{i}(h) \overline{a_{i}}\left[\overline{b_{i}}+\sum_{j=1}^{m} \overline{p_{i j}}\left(\alpha_{j}\left|x_{i_{0}}\right|+\left|f_{j}(0)\right|\right)+\overline{I_{i}}\right]\right\} \\
\geq & \frac{1}{\omega} \sum_{s=0}^{\omega-1} \xi_{i}(h) r_{1}\left(\underline{\gamma_{i}}-\overline{a_{i}} \sum_{j=1}^{m} \overline{p_{i j}} \alpha_{j}\right) \\
> & 0 .
\end{aligned}
$$

This contradicts (2.17).

(b) When $\left|y_{i_{0}}\right|>\left|x_{i_{0}}\right|$, we have

$$
\begin{aligned}
& \frac{\mu}{\omega} \sum_{s=0}^{\omega-1}\left\{\left(1-e^{-\frac{\Gamma_{j}^{*}(s)+\gamma_{j}^{*}(s)}{2} h}\right) y_{j_{0}}-\eta_{j}(h)\left\{\frac{\Gamma_{j}^{*}(s)+\gamma_{j}^{*}(s)}{2} y_{j_{0}}\right.\right. \\
& \left.-c_{j}\left(s, y_{j_{0}}\right)\left[d_{j}\left(s, y_{j_{0}}\right)-\sum_{i=1}^{k} q_{j i} g_{i}\left(x_{i}\right)+J_{j}(s)\right]\right\} \\
& +\frac{1-\mu}{\omega} \sum_{s=0}^{\omega-1}\left(1-e^{-\frac{\Gamma_{j}^{*}(s)+\gamma_{j}^{*}(s)}{2} h}\right) y_{j_{0}} \\
& \geq \frac{1}{\omega} \sum_{s=0}^{\omega-1}\left\{\left(1-e^{-\frac{\Gamma_{j}^{*}(s)+\gamma_{j}^{*}(s)}{2}}\right)\left|y_{j_{0}}\right|\right. \\
& \left.-\eta_{j}(h)\left[\frac{\Gamma_{j}^{*}(s)-\gamma_{j}^{*}(s)}{2}\left|y_{j_{0}}\right|-\overline{c_{j}}\left(\overline{d_{j}}+\sum_{i=1}^{k} \overline{q_{j i}}\left(\beta_{i}\left|x_{i_{0}}\right|+\left|g_{i}(0)\right|\right)+\overline{J_{j}}\right)\right]\right\} \\
& \geq \frac{1}{\omega} \sum_{s=0}^{\omega-1}\left\{\gamma_{j}^{*}(s) \eta_{j}(h)\left|y_{j_{0}}\right|-\eta_{j}(h) \overline{c_{j}}\left[\overline{d_{j}}+\sum_{i=1}^{k} \overline{q_{j i}}\left(\beta_{i}\left|x_{i_{0}}\right|+\left|g_{i}(0)\right|\right)+\overline{J_{j}}\right]\right\} \\
& \geq \frac{1}{\omega} \sum_{s=0}^{\omega-1}\left\{\underline{\gamma_{j}^{*}} \eta_{j}(h)\left|y_{j_{0}}\right|-\eta_{j}(h) \overline{c_{j}}\left[\overline{d_{j}}+\sum_{i=1}^{k} \overline{q_{j i}}\left(\beta_{i}\left|y_{j_{0}}\right|+\left|g_{i}(0)\right|\right)+\overline{J_{j}}\right]\right\}
\end{aligned}
$$




$$
\begin{aligned}
& \geq \frac{1}{\omega} \sum_{s=0}^{\omega-1} \eta_{j}(h) r_{2}\left(\underline{\gamma_{j}^{*}}-\overline{c_{j}} \sum_{i=1}^{k} \overline{q_{j i}} \beta_{i}\right) \\
& >0 .
\end{aligned}
$$

This contradicts (2.18).

Claim 2. $\left|y_{j_{0}}\right|<d+r_{2}$. Otherwise, $\left|y_{j_{0}}\right| \geq d+r_{2}$. We consider two possible cases: (a) $\left|x_{i_{0}}\right| \leq\left|y_{j_{0}}\right|$; (b) $\left|x_{i_{0}}\right|>\left|y_{j_{0}}\right|$. This arguments of (a) and (b) are similar to this arguments of (b) and (a) in Claim 1, then the proof is omitted. Hence Claim 2 holds. In view of Claim 1 and Claim 2, we have $\left|x_{i_{0}}\right|<d+r_{1}$ and $\left|y_{j_{0}}\right|<d+r_{2}$. Thus

$$
\|z\|=\sum_{i=1}^{k}\left|x_{i}\right|+\sum_{j=1}^{m}\left|y_{j}\right|<m\left(d+r_{2}\right)+k\left(d+r_{1}\right) .
$$

Hence $z \in \Omega \cap \mathbb{R}^{m+k}$. This contradicts the fact $z \in \partial \Omega \cap \mathbb{R}^{m+k}$. According to topological degree theory and by taking $J=I$ since $\operatorname{Ker} L=\operatorname{Im} Q$, we obtain,

$$
\begin{aligned}
& \operatorname{deg}\left\{-J Q N z, \Omega \cap \operatorname{Ker} L,(0,0)^{T}\right\} \\
= & \operatorname{deg}\left\{\phi\left(x_{1}, \ldots, x_{k}, y_{1}, \ldots, y_{m}, 1\right), \Omega \cap \operatorname{Ker} L,(0,0, \ldots, 0)^{T}\right\} \\
= & \operatorname{deg}\left\{\phi\left(x_{1}, \ldots, x_{k}, y_{1}, \ldots, y_{m}, 0\right), \Omega \cap \operatorname{Ker} L,(0, \ldots, 0)^{T}\right\} \\
= & \operatorname{deg}\left\{\left(p_{1} x_{1}, \ldots, p_{k} x_{k}, q_{1} y_{1}, \ldots, q_{m} y_{m}\right)^{T}, \Omega \cap \operatorname{Ker} L,(0,0, \ldots, 0)^{T}\right\} \\
\neq & 0 .
\end{aligned}
$$

So far, we have proved that $\Omega$ satisfies all the assumptions in Lemma 2.1. Therefore, the system (1.2) has at least one $\omega$-periodic solution.

\section{Global exponential stability of periodic solution}

It follows from Theorem 2.1 that the system (1.2) has at least a $\omega$-periodic solution $z^{*}(n)=\left(x_{1}^{*}(n), \ldots, x_{k}^{*}(n), y_{1}^{*}\left(n, \ldots, y_{m}^{*}(n)\right)^{T}\right.$. Obviously, if this periodic solution is globally exponentially stable, then it is unique. We next show that the periodic solution $z^{*}(n)$ is globally exponentially stable.

Assume that $z(n)=\left(x_{1}(n), \ldots, x_{k}(n), y_{1}(n), \ldots, y_{m}(n)\right)^{T}$ is an arbitrary solution of the system (1.2). Make a transformation for the system (1.2): $u_{i}(n)=x_{i}(n)-x_{i}^{*}(n), v_{j}(n)=y_{j}(n)-y_{j}^{*}(n)$, then the system (1.2) becomes

$$
\text { (3.1) } \begin{aligned}
& u_{i}(n+1) \\
= & e^{-\frac{\Gamma_{i}(n)+\gamma_{i}(n)}{2} h_{i}} u_{i}(n)+\xi_{i}(h)\left\{\frac{\Gamma_{i}(n)+\gamma_{i}(n)}{2} u_{i}(n)\right. \\
& -a_{i}\left(n, x_{i}(n)\right) b_{i}\left(n, x_{i}(n)\right)+a_{i}\left(n, x_{i}^{*}(n)\right) b_{i}\left(n, x_{i}^{*}(n)\right) \\
& -\sum_{j=1}^{m} p_{i j}(n)\left[a_{i}\left(n, x_{i}(n)\right) f_{j}\left(y_{j}\left(n-\tau_{i j}(n)\right)\right)\right.
\end{aligned}
$$




$$
\begin{aligned}
& \left.\left.-a_{i}\left(n, x_{i}^{*}(n)\right) f_{j}\left(y_{j}^{*}\left(n-\tau_{i j}(n)\right)\right)\right]-\left[a_{i}\left(n, x_{i}(n)\right)-a_{i}\left(n, x_{i}^{*}(n)\right)\right] I_{i}(n)\right\}, \\
& v_{j}(n+1) \\
= & e^{-\frac{\Gamma_{j}^{*}(n)+\gamma_{j}^{*}(n)}{2} h} v_{j}(n)+\eta_{j}(h)\left\{\frac{\Gamma_{j}^{*}(n)+\gamma_{j}^{*}(n)}{2} v_{j}(n)\right. \\
& -c_{j}\left(n, y_{j}(n)\right) d_{j}\left(n, y_{j}(n)\right)+c_{j}\left(n, y_{j}^{*}(n)\right) d_{j}\left(n, y_{j}^{*}(n)\right) \\
& -\sum_{i=1}^{k} q_{j i}(n)\left[c_{j}\left(n, y_{j}(n)\right) g_{i}\left(x_{i}\left(n-\sigma_{j i}(n)\right)\right)\right. \\
& \left.\left.-c_{j}\left(n, y_{j}^{*}(n)\right) g_{i}\left(x_{i}^{*}\left(n-\sigma_{j i}(n)\right)\right)\right]-\left[c_{j}\left(n, y_{j}(n)\right)-c_{j}\left(n, y_{j}^{*}(n)\right)\right] J_{j}(n)\right\} .
\end{aligned}
$$

Clearly, the periodic solution $z^{*}(n)$ of the system (1.2) is exponentially stable if and only if the equilibrium point $o$ of the system (3.1) is exponentially stable.

Theorem 3.1. Assume that $\left(H_{2}\right)-\left(H_{7}\right)$ hold. Further assume that

$$
\begin{aligned}
& \underline{\gamma_{i}}>\sum_{j=1}^{m} \overline{p_{i j}}\left(\overline{a_{i}} \alpha_{j}+\overline{L_{i}^{a}} M_{j}\right)+\overline{L_{i}^{a} I_{i}} ; \\
& \gamma_{j}^{*}>\sum_{i=1}^{k} \overline{q_{j i}}\left(\overline{c_{j}} \beta_{i}+\overline{L_{j}^{c}} N_{i}\right)+\overline{L_{j}^{c} J_{j}} .
\end{aligned}
$$

Then the system (1.2) has a unique periodic solution which is globally exponentially stable.

Proof. Since $\left(h_{2}\right)$ implies that $\left(h_{1}\right)$ in Theorem 2.1 holds, we only need to show that the equilibrium point $O$ of the system (3.1) is globally exponentially stable.

From the system (3.1), we obtain

$$
\begin{aligned}
& \left|u_{i}(n+1)\right| \\
& \leq e^{-\frac{\Gamma_{i}(n)+\gamma_{i}(n)}{2} h}\left|u_{i}(n)\right|+\xi_{i}(h)\left\{\frac{\Gamma_{i}(n)-\gamma_{i}(n)}{2}\left|u_{i}(n)\right|\right. \\
& +\sum_{j=1}^{m}\left|p_{i j}(n)\right| \mid a_{i}\left(n, x_{i}(n)\right) f_{j}\left(y_{j}\left(n-\tau_{i j}(n)\right)\right) \\
& \left.-a_{i}\left(n, x_{i}^{*}(n)\right) f_{j}\left(y_{j}^{*}\left(n-\tau_{i j}(n)\right)\right)|+| a_{i}\left(n, x_{i}(n)\right)-a_{i}\left(n, x_{i}^{*}(n)\right)|| I_{i}(n) \mid\right\} \\
& \leq e^{-\frac{\Gamma_{i}(n)+\gamma_{i}(n)}{2} h}\left|u_{i}(n)\right|+\xi_{i}(h)\left\{\frac{\Gamma_{i}(n)-\gamma_{i}(n)}{2}\left|u_{i}(n)\right|\right. \\
& +\sum_{j=1}^{m} \overline{p_{i j}}\left[\left|a_{i}\left(n, x_{i}(n)\right)-a_{i}\left(n, x_{i}^{*}(n)\right)\right|\right. \\
& \times \mid f_{j}\left(y _ { j } ( n - \tau _ { i j } ( n ) ) | + | a _ { i } \left(n, x_{i}^{*}(n)|| f_{j}\left(y_{j}\left(n-\tau_{i j}(n)\right)\right)\right.\right.
\end{aligned}
$$




$$
\begin{aligned}
& \left.-f_{j}\left(y_{j}^{*}\left(n-\tau_{i j}(n)\right) \mid\right]+\overline{L_{i}^{a}}\left|u_{i}(n)\right| \overline{I_{i}}\right\} \\
\leq & e^{-\frac{\Gamma_{i}(n)+\gamma_{i}(n)}{2}} h\left|u_{i}(n)\right|+\xi_{i}(h)\left\{\frac{\Gamma_{i}(n)-\gamma_{i}(n)}{2}\left|u_{i}(n)\right|\right. \\
& \left.+\sum_{j=1}^{m} \overline{p_{i j}}\left[\overline{L_{i}^{a}} M_{j}\left|u_{i}(n)\right|+\overline{a_{i}} \alpha_{j}\left|v_{j}\left(n-\tau_{i j}(n)\right)\right|\right]+\overline{L_{i}^{a}}\left|u_{i}(n)\right| \overline{I_{i}}\right\},
\end{aligned}
$$

$$
\begin{aligned}
& \left|v_{j}(n+1)\right| \leq e^{-\frac{\Gamma_{j}^{*}(n)+\gamma_{j}^{*}(n)}{2} h}\left|v_{j}(n)\right|+\eta_{j}(h)\left\{\frac{\Gamma_{j}^{*}(n)-\gamma_{j}^{*}(n)}{2}\left|v_{j}(n)\right|\right. \\
& \left.+\sum_{i=1}^{k} \overline{q_{j i}}\left[\overline{L_{j}^{c}} N_{i}\left|v_{j}(n)\right|+\overline{c_{j}} \beta_{j}\left|u_{i}\left(n-\sigma_{j i}(n)\right)\right|\right]+\overline{L_{j}^{c}}\left|v_{j}(n)\right| \overline{J_{j}}\right\} .
\end{aligned}
$$

Consider the functions $F_{i}(\cdot)$ and $G_{j}(\cdot)$ defined by

$$
\begin{aligned}
& F_{i}(x)=1-x+x \xi_{i}(h)\left\{\underline{\gamma_{i}}-\sum_{j=1}^{m} \overline{p_{i j}}\left(\overline{a_{i}} \alpha_{j} x^{\tau_{i j}(n)}+\overline{L_{i}^{a}} M_{j}\right)-\overline{L_{i}^{a} I_{i}}\right\}, \\
& G_{j}(y)=1-y+y \eta_{j}(h)\left\{\underline{\gamma_{j}^{*}}-\sum_{i=1}^{k} \overline{q_{j i}}\left(\overline{c_{j}} \beta_{i} y^{\sigma_{j i}(n)}+\overline{L_{j}^{c}} N_{i}\right)-\overline{L_{j}^{c} J_{j}}\right\} .
\end{aligned}
$$

Since we have for $i=1, \ldots, k, j=1, \ldots, m$,

$$
F_{i}(1)=\xi_{i}(h)\left\{\underline{\gamma_{i}}-\sum_{i=1}^{k} \overline{p_{i j}}\left(\overline{a_{i}} \alpha_{i}+\overline{L_{i}^{a}} M_{j}\right)-\overline{L_{i}^{a} I_{i}}\right\}>0
$$

and

$$
G_{i}(1)=\eta_{j}(h)\left\{\underline{\gamma_{j}^{*}}-\sum_{i=1}^{k} \overline{q_{j i}}\left(\overline{c_{j}} \beta_{i}+\overline{L_{j}^{c}} N_{i}\right)-\overline{L_{j}^{c} J_{j}}\right\}>0,
$$

then there must be a real number $1<\eta<+\infty$ such that for $i=1, \ldots, k$, $j=1, \ldots, m$,

$$
F_{i}(\eta)>0, \quad G_{j}(\eta)>0 .
$$

Therefore, we have for $i=1, \ldots, k, j=1, \ldots, m$,

$$
\begin{aligned}
& \eta-\eta \xi_{i}(h)\left\{\underline{\gamma_{i}}-\sum_{j=1}^{m} \overline{p_{i j}}\left(\overline{a_{i}} \eta^{\tau_{i j}(n)} \alpha_{j}+\overline{L_{i}^{a}} M_{j}\right)-\overline{L_{i}^{a} I_{i}}\right\}<1, \\
& \eta-\eta \eta_{j}(h)\left\{\underline{\gamma_{j}^{*}}-\sum_{i=1}^{k} \overline{q_{j i}}\left(\overline{c_{j}} \eta^{\sigma_{j i}(n)} \beta_{i}+\overline{L_{j}^{c}} N_{i}\right)-\overline{L_{j}^{c} J_{j}}\right\}<1 .
\end{aligned}
$$

Now let we consider nonnegative sequence $U_{i}(n), V_{j}(n)$ for $i=1, \ldots, k, j=$ $1, \ldots, m, n \in \mathbb{Z}$, defined by

$$
U_{i}(n)=\eta^{n}\left|u_{i}(n)\right|, \quad V_{j}(n)=\eta^{n}\left|v_{j}(n)\right| .
$$


Thus from (3.2), (3.3) and ((3.6), we have

$$
\begin{aligned}
& U_{i}(n+1) \\
\leq & \eta e^{-\frac{\Gamma_{i}(n)+\gamma_{i}(n)}{2} h}\left|U_{i}(n)\right|+\xi_{i}(h) \eta\left\{\frac{\Gamma_{i}(n)-\gamma_{i}(n)}{2}\left|U_{i}(n)\right|\right. \\
& \left.+\sum_{j=1}^{m} \overline{p_{i j}}\left[\overline{L_{i}^{a}} M_{j}\left|U_{i}(n)\right|+\alpha_{j} \overline{a_{i}} \eta^{\tau_{i j}(n)}\left|V_{j}\left(n-\tau_{i j}(n)\right)\right|\right]+\overline{L_{i}^{a} I_{i}}\left|U_{i}(n)\right|\right\}, \\
(3.8) \quad & V_{j}(n+1) \\
\leq & \eta e^{-\frac{\Gamma_{j}^{*}(n)+\gamma_{j}^{*}(n)}{2} h}\left|V_{j}(n)\right|+\eta_{j}(h) \eta\left\{\frac{\Gamma_{j}^{*}(n)-\gamma_{j}^{*}(n)}{2}\left|V_{j}(n)\right|\right. \\
& \left.+\sum_{i=1}^{k} \overline{q_{j i}}\left[\overline{L_{j}^{c}} N_{i}\left|V_{j}(n)\right|+\beta_{i} \overline{c_{j}} \eta^{\sigma_{j i}(n)}\left|U_{i}\left(n-\sigma_{j i}(n)\right)\right|\right]+\overline{L_{j}^{c} J_{j}}\left|V_{j}(n)\right|\right\} .
\end{aligned}
$$

Denote

$$
M=\eta^{n} \max _{1 \leq i \leq k, 1 \leq j \leq m}\left\{\sup _{l \in(-\infty, 0]_{\mathbb{Z}}}\left|u_{i}(l)\right|, \sup _{l \in(-\infty, 0]_{\mathbb{Z}}}\left|v_{j}(l)\right|\right\}, M>0 .
$$

It follows that from (3.6) and the definition of the constant $M$ that

$$
U_{i}(l) \leq M, \quad V_{j}(l) \leq M
$$

for $i=1, \ldots, k, j=1, \ldots, m, l \in(-\infty, 0]_{\mathbb{Z}}$.

We claim that for $i=1, \ldots, k, j=1, \ldots, m, n \in \mathbb{Z}_{0}^{+}$,

$$
U_{i}(n) \leq M, \quad V_{j}(n) \leq M
$$

Suppose that claim (3.9) is not valid in sense, then there exist a $k^{*}$-th component among $U_{i}(\cdot)$ and a first time $n_{1} \in \mathbb{Z}^{+}$, such that $U_{k^{*}}(n)>M$ and $U_{i}(n) \leq M$ for $n \in\left(-\infty, n_{1}-1\right]_{\mathbb{Z}}$, while $U_{i}(n) \leq M$ for $i=1, \ldots, k, i \neq k^{*}, n \in$ $\left(-\infty, n_{1}\right]_{\mathbb{Z}}, V_{j}(n) \leq M$ for $j=1, \ldots, m, n \in\left(-\infty, n_{1}\right]_{\mathbb{Z}}$; or there exist a $k^{*}$-th component among $V_{j}(\cdot)$ and a first time $n_{1} \in \mathbb{Z}^{+}$, such that $V_{k^{*}}(n)>M$ and $V_{j}(n) \leq M$ for $n \in\left(-\infty, n_{1}-1\right]_{\mathbb{Z}}$, while $V_{j}(n) \leq M$ for $j=1, \ldots, m, j \neq$ $k^{*}, n \in\left(-\infty, n_{1}\right]_{\mathbb{Z}}, U_{i}(n) \leq M$ for $i=1, \ldots, k, n \in\left(-\infty, n_{1}\right]_{\mathbb{Z}}$. If the first case holds, then from (3.4) and (3.7), we obtain

$$
\begin{aligned}
M< & U_{k}\left(n_{1}\right) \\
\leq & \left\{\eta e^{-\frac{\Gamma_{i}\left(n_{1}\right)+\gamma_{i}\left(n_{1}\right)}{2} h}+\eta \xi_{i}(h)\left[\frac{\Gamma_{i}\left(n_{1}\right)-\gamma_{i}\left(n_{1}\right)}{2}\right.\right. \\
& \left.\left.\quad+\sum_{j=1}^{m} \overline{p_{i j}}\left(\alpha_{j} \overline{a_{i}} \eta^{\tau_{i j}\left(n_{1}\right)}+\overline{L_{i}^{a}} M_{j}\right)+\overline{L_{i}^{a} I_{i}}\right]\right\} M \\
\leq & \left\{\eta-\eta \xi_{i}(h)\left[\underline{\gamma_{i}}-\sum_{j=1}^{m} \overline{p_{i j}}\left(\alpha_{j} \overline{a_{i}} \eta^{\tau_{i j}\left(n_{1}\right)}+\overline{L_{i}^{a}} M_{j}\right)-\overline{L_{i}^{a} I_{i}}\right]\right\} M \\
\leq & M,
\end{aligned}
$$


which is a contradiction. If the second case holds, a contradiction also can be led from (3.5) and (3.8). Thus the claim (3.9) is valid. From (3.6) and (3.9) we deduce that

$$
\left|u_{i}(n)\right| \leq\left(\frac{1}{\eta}\right)^{n} M, \quad\left|v_{j}(n)\right| \leq\left(\frac{1}{\eta}\right)^{n} M
$$

for $i=1, \ldots, k, j=1, \ldots, m, n \in \mathbb{Z}_{0}^{+}$. Since the constant $\eta$ satisfies $1<\eta$ and the initial values are arbitrary, we conclude from the above that the equilibrium o is globally exponentially stable. That is, the periodic solution $z^{*}(n)$ of the system (1.2) is globally exponentially stable. The proof is complete.

\section{An example}

Example 1. Consider the following second-order discrete-time Cohen-Grossberg bidirectional associative memory neural networks with delays:

(4.1)

$$
\left\{\begin{aligned}
& x_{1}(n+1) \\
= & x_{1}(n) e^{-\frac{42+7 \sin 36 \pi n+56+7 \sin 36 \pi n}{2} h}+\xi_{1}(h)\left\{\frac{42+7 \sin 36 \pi n+56+7 \sin 36 \pi n}{2} x_{1}(n)\right. \\
& -\left[7+\sin 36 \pi n-\frac{\cos 36 \pi n}{1+x_{1}^{2}(n)}\right] \\
& \left.\times\left[7 x_{1}(n)-\left(\frac{3}{80}+\frac{1}{16} \sin 36 \pi n\right) \sin y_{1}(n-(2+\sin 36 \pi n))+\sin 36 \pi t\right]\right\}, \\
& y_{1}(n+1) \\
= & y_{1}(n) e^{-\frac{38+42}{2} h}+\eta_{1}(h)\left\{\frac{38+42}{2} y_{1}(n)-\left[5-\frac{\sin 36 \pi n}{4+y_{1}^{2}(n)}\right]\right. \\
& \left.\times\left[8 y_{1}(n)-\left(\frac{1}{50}+\frac{2}{25} \cos 36 \pi n\right) \cos x_{1}(n-(2+\sin 36 \pi n))+\cos 36 \pi n\right]\right\},
\end{aligned}\right.
$$

where

$$
n>3, \xi_{1}(h)=\frac{1-e^{-\frac{42+7 \sin 36 \pi n+56+7 \sin 36 \pi n}{2} h}}{\frac{42+7 \sin 36 \pi n+56+7 \sin 36 \pi n}{2}}, \eta_{1}(h)=\frac{1-e^{-\frac{38+42}{2} h}}{\frac{38+42}{2}} .
$$

Since the value of the activation functions at zero is not zero, then the results in [4] can not ensure the global exponential stability of the system (4.1). However, by using Theorem 3.1, we can prove the periodic solution of the system (4.1) is globally exponentially stable. Since for $n>3, x, y \in \mathbb{R}$,

$$
\begin{aligned}
\left|a_{1}(n, x)-a_{1}(n, y)\right| & =\left|\frac{\cos 36 \pi t}{1+x^{2}}-\frac{\cos 36 \pi t}{1+y^{2}}\right| \\
& \leq|x-y| \frac{|x+y|}{\left(1+x^{2}\right)\left(1+y^{2}\right)} \\
& \leq|x-y|\left(\frac{|x|}{1+x^{2}}+\frac{|y|}{1+y^{2}}\right) \\
& \leq|x-y|, \\
\left|c_{1}(n, x)-c_{1}(n, y)\right| & \leq \frac{|x-y|}{2},
\end{aligned}
$$




$$
\begin{aligned}
& \frac{a_{1}(n, x) b_{1}(n, x)-a_{1}(n, y) b_{1}(n, y)}{x-y} \\
= & 7(7+\sin 36 \pi t)-7 \cos 200 \pi t \frac{1-x y}{\left(1+x^{2}\right)\left(1+y^{2}\right)} \\
\geq & 7(7+\sin 36 \pi t)-7 \frac{1+|x y|}{\left(1+x^{2}\right)\left(1+y^{2}\right)} \\
\geq & 42+7 \sin 36 \pi t, \\
& \frac{a_{1}(t, x) b_{1}(t, x)-a_{1}(t, y) b_{1}(t, y)}{x-y} \\
= & 7(7+\sin 36 \pi t)-7 \cos 200 \pi t \frac{1-x y}{\left(1+x^{2}\right)\left(1+y^{2}\right)} \\
\leq & 7(7+\sin 36 \pi t)+7 \frac{1+|x y|}{\left(1+x^{2}\right)\left(1+y^{2}\right)} \\
\leq & 56+7 \sin 36 \pi t, \\
38 \leq & \frac{c_{1}(t, x) d_{1}(t, x)-c_{1}(t, y) d_{1}(t, y)}{x-y} \leq 42,
\end{aligned}
$$

therefore, in Theorem 3.1.

$$
\begin{array}{r}
m=n=1, L_{1}^{a}=1, L_{1}^{c}=\frac{1}{2}, \underline{\gamma_{1}}=35, \underline{\gamma_{1}^{*}}=38, M_{1}=1, N_{1}=1, \\
\alpha_{1}=1, \beta_{1}=1, \overline{a_{1}}=8, \overline{c_{1}}=6, \omega=\frac{1}{18}, \overline{I_{1}}=\overline{J_{1}}=1, \overline{p_{11}}=0.1, \overline{q_{11}}=0.1 .
\end{array}
$$

It is easy to show that the rest conditions in Theorem 3.1 are also satisfied. Hence by Theorem 3.1, the periodic solution of the system (4.1) is globally and exponentially stable.

\section{Conclusions}

By employing coincidence degree theory and using Halanay-type inequality technique, a sufficient condition is given to guarantee the existence and global exponential stability of periodic solutions for the two-dimensional discrete-time Cohen-Grossberg BAM neural networks. Compared with the results in existing papers, in our result on the existence of periodic solution, the boundedness conditions on the activation functions are replaced with global Lipschitz conditions. In our result on the existence and global exponential stability of periodic solution, the assumptions in existing papers that the value of activation functions at zero is zero are removed.

Acknowledgements. The authors would like to thank the reviewers and the editor for their valuable suggestions and comments which have led a much improved paper. 


\section{References}

[1] S. Arik and Z. Orman, Global stability analysis of Cohen-Grossberg neural networks with time varying delays, Phys. Lett. A 341 (2005), no. 5-6, 410-421.

[2] J. Cao and J. Liang, Boundedness and stability of Cohen-Grossberg neural networks with time-varying delays, J. Math. Anal. Appl. 296 (2004), no. 2, 665-685.

[3] A. P. Chen and J. D. Cao, Periodic bi-directional Cohen-Grossberg neural networks with distributed delays, Nonlinear Anal. 66 (2007), no. 12, 2947-2961.

[4] A. P. Chen and F. L. Chen, Periodic solution to BAM neural network with delays on time scalesNeurocomputing 73 (2009), no. 1-3, 274-282.

[5] A. P. Chen, L. H. Huang, Z. G. Liu, and J. D.Cao, Periodic bidirectional associative memory neural networks with distributed delaysJ. Math. Anal. Appl. 317 (2006), no. 1, 80-102.

[6] T. Chen and L. Rong, Delay-independent stability analysis of Cohen-Grossberg neural networks, Phys. Lett. A 317 (2003), no. 5-6, 436-449.

[7] Z. Chen, D. H. Zhao, and X. L. Fu, Discrete analogue of high-order periodic CohenGrossberg neural networks with delays, Appl. Math. Comput. 214 (2009), no. 1, 210-217.

[8] M. Cohen and S. Grossberg, Absolute stability and global pattern formation and parallel memory storage by competitive neural networks, IEEE Trans. Systems Man Cybernet. 13 (1983), no. 5, 815-826.

[9] B. T. Cui and W. Wu, Global exponential stability of Cohen-Grossberg neural networks with distributed delays, Neurocomputing 72 (2008), no. 1-3, 386-391.

[10] J. Feng and S. Xu, New criteria on global robust stability of Cohen-Grossberg neural networks with time varying delays, Neurocomputing 72 (2008), no. 1-3, 445-457.

[11] J. Feng, S. Y. Xu, and Y. Zou, Delay-dependent stability of neutral type neural networks with distributed delays, Neurocomputing 72 (2009), 2576-2580.

[12] R. E. Gaines and J. L. Mawhin, Coincidence Degree, and Nonlinear Differential Equations, Springer Berlin, 1977.

[13] Y. Li, Existence and stability of periodic solutions for Cohen-Grossberg neural networks with multiple delays, Chaos Solitons Fractals 20 (2004), no. 3, 459-466.

[14] Y. K. Li, Global stability and existence of periodic solutions of discrete delayed cellular neural networks, Phys. Lett. A 333 (2004), no. 1-2, 51-61.

[15] X. Liao, C. Li, and K. Wong, Criteria for exponential stability of Cohen-Grossberg neural networks, Neural Networks 17 (2004), no. 10, 1401-1414.

[16] J. Liang and J. Cao, Exponential stability of continuous-time and discrete-time bidirectional associative memory networks with delays, Chaos Solitons Fractals 22 (2004), no. $4,773-785$.

[17] L. Liang, J. Cao, and D. Ho, Discrete-time bidirectional associative memory neural networks with variable delays, Phys. Lett. A 335 (2005), 226-234.

[18] J. A. Liu and G. D. Zong, New delay-dependent asymptotic stability conditions concerning BAM neural networks of neutral type, Neurocomputing 72 (2009), 2549-2555.

[19] W. L. Lu and T. P. Chen, $R_{+}^{n}$ global stability of a Cohen-Grossberg network system with nonnegative equilibria, Neural Networks 20 (2007), no. 6, 714-722.

[20] K. N. Lu, D. Y. Xu, and Z. C. Yang, Global attraction and stability for Cohen-Grossberg neural networks with delays, Neural Networks 19 (2006), no. 10 1538-1549.

[21] S. Mohamad and K. Gopalsamy, Dynamics of a class of discrete time neural networks and their continuous time counterparts, Math. Comput. Simulation 53 (2000), no. 1-2, $1-39$.

[22] _ Exponential stability of continuous-time and discrete-time cellular neural networks with delays, Appl. Math. Comput. 135 (2003), no. 1, 17-38.

[23] S. Mohamad and A. G. Naim, Discrete-time analogues of integrodifferential equations modelling bidirectional neural networks, J. Comput. Appl. Math. 138 (2002), no. 1, 1-20. 
[24] Ju H. Park, Robust stability of bidirectional associative memory neural networks with time delays, Phys. Lett. A 349 (2006), no. 6, 494-499.

[25] Ju H. Park and O. M. Kwon, Delay-dependent stability criterion for bidirectional associative memory neural networks with interval time-varying delays, Modern Physics Letters B 23 (2009), no. 1, 35-46.

[26] Ju H. Park, S. M. Lee, and O. M. Kwon, On exponential stability of associative memory neural networks with time-varying delays, Chaos Solitons Fractals 39 (2009), no. 3, 1083-1091.

[27] Ju H. Park, C. H. Park, O. M. Kwon, and S. M. Lee, A new stability criterion for bidirectional associative memory neural networks of neutral type, Appl. Math. Comput. 199 (2008), no. 2, 716-722.

[28] L. B. Rong and T. P. Chen, New results on the robust stability of Cohen-Grossberg neural networks with delays, Neural Process Lett. 24 (2006), no. 3, 193-202.

[29] Q. K. Song and J. D. Cao, Robust stability in Cohen-Grossberg neural networks with both time-varying and distributed delays, Neural Process Lett. 27 (2008), no. 2, 179-196.

[30] C. Sun and C. B. Feng, Discrete-time analogues of intergrodifferential equations modelling neural networks, Phys. Lett. A 334 (2005), 180-191.

[31] J. Sun and L. Wang, Global exponential stability and periodic solutions of CohenGrossberg neural networks with continuously distributed delays, Phys. D 208 (2005), no. 1-2, 1-20.

[32] L. Wang, Stability of Cohen-Grossberg neural networks with distributed delays, Appl. Math. Comput. 160 (2005), no. 1, 93-110.

[33] Z. D. Wang, Y. R. Liu, and M. Li, Stability analysis for stochastic Cohen-Grossberg neural networks with mixed time delays, IEEE Trans. Neural Networks 17 (2006), no. $3,814-820$

[34] Z. D. Wang, Y. R. Liu, and X. H. Liu, On global asymptotic stability of neural networks with discrete and distributed delays, Phys Lett. A 345 (2005), no. 4-6, 299-308.

[35] W. Wu, B. T. Cui, and X. Y. Lou, Some criteria for asymptotic stability of CohenGrossberg neural networks with time varying delays, Neurocomputing 70 (2007), no. 4-6, 1085-1088.

[36] W. Xiong and J. Cao, Global exponential stability of discrete-time Cohen-Grossberg neural networks, Neurocomputing 64 (2005), 433-446.

[37] H. J. Xiang and J. D. Cao, Exponential stability of periodic solution to Cohen-Grossberg type BAM neural networks with time-varying delays, Neurocomputing $\mathbf{7 2}$ (2009), no. 7-8, 1702-1711.

[38] W. W. Yu, J. D. Cao, and J. Wang, An LMI approach to global asymptotic stability of the delayed Cohen-Grossberg neural networks via nonsmooth analysis, Neural Networks 20 (2007), no. 7, 810-818.

[39] Z. G. Zeng, J.W, Global exponential stability of recurrent networks with time-varying delays in the presence of strong external stimuli, Neural Networks 19 (2006), no. 10, $1528-1537$.

[40] J. Zhang, Y. Suda, and H. Komine, Global exponential stability of Cohen-Grossberg neural networks with variable delays, Phys. Lett. A 338 (2005), no. 1, 44-50.

[41] Z. Q. Zhang and D. M. Zhou, Existence and global exponential stability of a periodic solution for a discrete-time interval general BAM neural networks, J. Franklin Inst. $\mathbf{3 4 7}$ (2010), no. 5, 763-780.

[42] H. Y. Zhao and L. Wang, Hopf bifucation in Cohen-Grossberg neural network with discrete delays, Nonlinear Anal.: Real World Appli. 8 (2007), no. 1, 73-89.

[43] H. Zhao, L. Sun, and G. Wang, Periodic oscillation of discrete-time bidirectional associative memory neural networks, Neurocomputing 70 (2007), 2924-2930. 
[44] T. Zhou, Y. Liu, and Y. C. Liu, Existence and global exponential stability of periodic solution for discrete time BAM neural networks, Appl. Math. Comput. 182 (2006), no. $2,1341-1354$.

[45] Z. Orman and S. Arik, New results for global stability of Cohen-Grossberg neural networks with multiple time delays, Neurocomputing 71 (2008), no. 16-18, 3053-3063.

ZHENGQIU ZHANG

College of Mathematics

HunAN University

Changsha 410082, P. R. China

E-mail address: zhangzhengqiu@hnu.cn

LIPING WANG

College of Mathematics

HunAN UNIVERSITY

Changsha 410082, P. R. China

E-mail address: wliping001@163.com 